first increases and then decreases, the flocculation value with $\mathrm{KCl}$ shows a continuous decrease.

4. The charge increases on dilution of the colloid, the increase in charge of a sol dialysed for 2 days being greater than of one dialysed for 10 days-the dilutions in the two cases being the same.

In a paper which has been sent for publication the above results have been utilised to show that in the case of colloidal ferric hydroxide some of the fundamental assumptions made by colloid chemists in explaining their results of coagulation of colloids by electrolytes, namely, that (1) the charge and stability go hand in hand, (2) the charge continuously decreases with the progress of dialysis, and (3) the applicability of the general dilution rule, are justified. The abnormality in any particular case has been explained as being due to the preferential adsorption of the stabilising ions which may be present in an amount greater than that which corresponds to the maximum in the cataphoretic speed-concentration curve of the colloid with the particular electrolyte.

B. N. Desar.

P. M. Barve.

Physical Chemistry Laboratory,

Wilson College, Bombay 7, India, Sept. 25.

Kolloidchem. Beihefte, 26, 384 ; 1928.

2 NATURE, 127, 666; 1931.

\section{Application of the Simonis Reaction to Monohydric Phenols.}

IN continuation of the work of Robertson, Sandrock, and Hendry ${ }^{1}$ on the condensation of monohydric phenols with esters of acylacetic acids by means of phosphoric oxide, the following results have been obtained : o-cresol, o-chlorophenol, o-bromophenol, $p$-chlorophenol, $p$-bromophenol, and $\beta$-napthol on submission to this reaction give rise to $1: 4$-pyrones. Compared with polyhydric and with monohydric phenols, the behaviour of $m$-cresol is interesting. On condensation with ethyl acetoacetate in the presence of phosphoric oxide this phenol yields $4: 7$-dimethylcoumarin, but with esters mono-substituted in the a-position 1:4-pyrones are obtained, for example, ethyl a-methylacetoacetate gives rise to $2: 3: 5$-trimethyl1 : 4-benzopyrone.

In view of the recent communication of Chakravarti on the application of the Simonis reaction to resorcinol (compare Canter, Curd, and Robertson ${ }^{3}$ ), it may be stated that the proof of the true nature of the products was first described by Canter in a dissertation submitted in 1930 for the degree of M.Sc. of the University of Lorrdon.

London School of Hygiene and

A. Robertson.

Tropical Medicine,

University of London,

$$
\text { Oct. } 10 .
$$

¿ Jour. Chem. Soc., p. 2426; 1931.

2 Jour. Ind. Chem. Soc., 8, 129; 1931.

- Jour. Chem. Sor., p. $1255 ; 1931$.

Aitken Gondensation Nuclei.

Investigations made by Prof. J. J. Nolan ${ }^{1}$ and collaborators, ${ }^{2,3,4}$ as well as Schweidler, ${ }^{5}$ Hess, ${ }^{6}$ Israel, ${ }^{7}$ Wait, ${ }^{8}$ and others, point to the importance of the part played by Aitken nuclei of condensation in atmospheric electric processes. It has been shown ${ }^{2}$ that some of these nuclei carry single electronic charges, the remainder being uncharged. It has been claimed $^{2}$ on theoretical grounds that, under equilibrium conditions, the ratio of charged to uncharged nuclei is substantially a constant.

At the Commonwealth Solar Observatory, situated on Mount Stromlo, ten miles from Canberra, the nuclei have been classified into groups according to their mobilities. To effect this, electrostatic fields have been applied to a current of air passing through a cylindrical condenser and the resultant diminution in nuclei content has been measured with a modification of the original Aitken counter. ${ }^{9}$

Observations, made near the open window of an atmospheric electric hut, gave as a mean of 49 tests :

\begin{tabular}{|ll|c|c|c|}
\hline \multicolumn{2}{|c|}{ Nuclei. } & & $\begin{array}{c}\text { Mobility, } \\
\text { cm./sec./volt./cm. }\end{array}$ & $\begin{array}{c}\text { Number } \\
\text { per c.c. }\end{array}$ \\
\cline { 1 - 2 } Intermediate ions ${ }^{10} N_{\mathrm{A}}$ & $\cdot$ & 0.005 & 975 \\
Large ions & $N_{\mathrm{B}}$ & $\cdot$ & $0.005-0.0005$ & 385 \\
Uncharged & $N_{0}$ & $\cdot$ & 0.0005 & 810 \\
\hline
\end{tabular}

Subsidiary tests indicated that the atmospheric small ion did not act as a nucleus in the apparatus, nor did any appreciable fraction of charged nuclei escape precipitation. The results appear to be statistically represented by the equation

$$
2 N_{0}=1.12 N_{\mathrm{A}}+1.37 N_{\mathrm{B}} \text {, }
$$

which does not indicate a constant ratio of charged to uncharged nuclei. The form of this equation was derived theoretically on the assumption that the uncharged nuclei were of two grades, $N_{a}$ and $N_{\beta}$, the former giving rise, by combination with atmospheric small ions, to intermediate ions $N_{A}$, and the latter to large ions $N_{B}$. It has been assumed that the small ions were the agents in distributing the charges amongst the nuclei, and any action of the intermediate and large ions was negligible. The above equation indicates that on the average the specific rate of the combination of the $N_{\mathrm{A}}$ nuclei with small ions of opposite sign is 1.12 times greater than that of the small ions with the $N_{\alpha}$ nuclei, whilst for the $N_{\mathrm{B}}: N_{\beta}$ equilibrium the corresponding value is $1 \cdot 37$. The average ratio of $N_{\alpha}: N_{\beta}$ deduced from these observations is $2: 1$.

Comparison of the $N_{\mathrm{A}}$ counts obtained by the Aitken counter with ion counts given by a Wulf unifilar electrometer attached to a cylindrical con. denser indicates that the $N_{A}$ group of nuclei carry single electronic charges. The mean of twenty observations gives the charge as $1 \cdot 0 \pm 0 \cdot 04$ electronic units.

Further work is proceeding in order to discover whether an equilibrium exists between $N_{\alpha}$ and $N_{\beta}$.

Commonwealth Solar Observatory, A. R. HogG.

Mount Stromlo, Canberra, F.C.T., Australia, Sept. 15.

1 NATURE, 113, $493 ; 1924$

Proc. Roy. Irish Acad., 37, 1 ; 1925.

Proc. Roy. Irish Acad., 38, 1 ; 1928-29.

- Ger. Beit. zur Geophys., 25, 414; 1930.

Wien. Ber., IIa (133), $29 ; 1924$.

Wien. Ber., IIa (138), 169 ; 1929.

Ger. Beit. zur Geophys., 26, 283 ; 1930.

Terr. Mag., 36, 111; 1931 .

- Proc. Roy. Soc. Edin., 38; 1890-91.

10 J. Pollock, Phil. Mag., 29, 514 and $636 ; 1915$.

11 Langevin, Compt. rend., 140, 305 ; 1907.

The Relativistic Opacity Goefficient.

THE recent paper of Prof. Milne ${ }^{1}$ on " The Analysis of Stellar Structure" has excited great interest, and several papers by various investigators have appeared on the subject. The knowledge of the opacity coefficient for the different physical states in which matter can exist in the stellar interiors is of importance in these developments. The value of the opacity coefficient for the non-relativistic non-degeneratecase was first worked out by Eddington following Kramers' treatment of X-ray absorption. The non-relativistie degenerate case has been recently treated by several authors.

No. 3239 , VoL. 128] 